Trauma Berufskrankh $2006 \cdot 8$ [Suppl 1]: S90-S92 DOI 10.1007/s10039-006-1109-z

Online publiziert: 16. Februar 2006

๑) Springer Medizin Verlag 2006

D. Haack · Stuttgart

\title{
Physiotherapie nach Verletzung
}

\author{
Kosten-Nutzen-Abwägung: Wie \\ lange soll Physiotherapie verordnet \\ werden? - Meinung 2
}

sind gängige Schlagworte, um die medizinischen Aktivitäten bürokratisch einzuschränken. Die ärztliche Behandlung wird weitgehend von äußeren Zwängen bestimmt.

\section{Gesetzliche Krankenkasse - gesetzliche Unfallversicherung}

Zunehmenden werden auch im GKV-System Leitlinien eingesetzt, um mengenmäßige Vorgaben einzuführen. Nicht mehr die medizinische Notwendigkeit ist der Maßstab der ärztlichen Aktivitäten, sondern die rigiden Vorgaben eines höchst unsozialen Gesetzes.

Für die niedergelassenen Ärzte kommt im GKV-System der Druck von der Kassenärztlichen Vereinigungen oder den Krankenkassen. Die Berufsgenossenschaften sind derzeit noch weitgehend außerhalb dieses Bereichs, auch aufgrund der kurzzeitigen Kontrollmöglichkeiten, die im GKV-System auf diese Weise nicht durchführbar sind. Aber auch für die UV-Träger hat sich $\mathrm{m}$. E. die Situation in Deutschland verändert durch:

- Rückgang der Beiträge für die UVTräger

- Hohe Arbeitslosenzahl mit Reduzierung der Höhe der Beitragseingänge

- Zunehmende ökonomische Betrachtung der Behandlungsverfahren

Damit ergeben sich folgende Fragen:
- Ist in der GUV eine Mengenbegrenzung wie im GKV-System notwendig?

- Müssen in der GUV Verordnungsvorgaben unter Leitliniengesichtspunkten eingeführt werden?

\section{Gesetzliche Grundlagen}

In $\$_{34}$ SGB VII ist festgelegt:

... die Kassenärztlichen Bundesvereinigungen haben gegenüber den UV und deren Verbänden die Gewähr dafür zu übernehmen, dass die Durchführung der Heilbehandlung den gesetzlichen und vertraglichen Erfordernissen entspricht.

\section{Richtlinien}

Der gemeinsame Bundesausschuss hat am 16.03.2004 so genannte Heilmittelrichtlinien verfasst. Sie dienen der Sicherung einer nach den Regeln der ärztlichen Kunst und unter Berücksichtigung des allgemein anerkannten Standes der medizinischen Erkenntnisse ausreichenden, zweckmäßigen und wirtschaftlichen Versorgung der Versicherten mit Heilmitteln. Damit ist gemeint, dass der allgemeiner Stand der Medizin

- ausreichend,

- zweckmäßig und

- wirtschaftlich

sein soll. Das heißt aber umgekehrt, dass jeder, der darüber hinaus verordnet, den allgemeinen Stand der Medizin verlässt, 
also unwirtschaftlich und unzweckmäBig handelt.

Die Richtlinien sind für die an der vertragsärztlichen Versorgung teilnehmenden Ärztinnen und Ärzte, Kassenärztlichen Vereinigungen, Krankenkassen und deren Verbände verbindlich. Die Spitzenverbände der Krankenkassen werden in den Rahmenempfehlungen über die einheitliche Versorgung mit Heilmitteln und Verträgen nach $\$ 125$ SGB V den in diesen Richtlinien beschriebenen Leistungsrahmen nicht überschreiten. Festgelegt wurden in ihnen:

- die Indikation

- die Art der verordnungsfähigen Heilmittel

- die Menge verordnungsfähiger Heilmittel in der Diagnosegruppe

- die Besonderheiten wie Wiederholungsverordnungen

Den Heilmittelverordnungen liegt in den jeweiligen Abschnitten des Heilmittelkatalogs ein definierter Regelfall zugrunde. Dieser geht von der Vorstellung aus, dass mit dem der Indikation zugeordneten Heilmittel im Rahmen der Gesamtverordnungsmenge des Regelfalls das angestrebte Therapieziel erreicht werden kann. Die Gesamtverordnungsmenge und die Anzahl der Behandlungen je Verordnung im Regelfall ergeben sich aus dem Heilmittelkatalog, in welchem Einzeldiagnosen zu Diagnosengruppen zusammengefasst sind. Die Verordnungsmenge richtet sich dabei nach den medizinischen Erfordernissen, nicht jede Schädigung bedarf der Höchstverordnungsmenge.

Eine Heilmittelverordnung im Regelfall liegt dann vor, wenn die Auswahl zwischen den möglichen, im Katalog angegebenen Heilmitteln getroffen wurde und die dort festgelegten Verordnungsmengen je Diagnosegruppe nicht überschritten werden. Rezidive oder neue Erkrankungsphasen können die Verordnung von Heilmitteln als erneuten Regelfall auslösen, wenn nach einer Heilmittelanwendung ein behandlungsfreies Intervall von 12 Wochen abgelaufen ist. Hierzu bestehen Ausnahmen: Lässt sich die Behandlung mit der Gesamtverordnungsmenge nicht abschließen, sind weitere Verordnungen außerhalb des Regelfalls möglich. Diese sind zu begründen und der zustän-

Trauma Berufskrankh 2006 - 8 [Suppl 1]: S90-S92 DOI 10.1007/s10039-006-1109-z

(c) Springer Medizin Verlag 2006

D. Haack

\section{Physiotherapie nach Verletzung. Kosten-Nutzen-Abwägung: Wie lange soll Physiotherapie verordnet werden? - Meinung 2}

\section{Zusammenfassung}

Auch seitens der gesetzlichen Unfallversicherungen wird überlegt, wie die verfügbaren Mittel gezielter und effizienter eingesetzt werden können. Dies ist unter dem Eindruck rückläufiger Beiträge, den hohen Arbeitslosenzahlen, der zunehmenden Zahl von Geschäftsaufgaben und den daraus resultierenden Mindereinnahmen zwingend geboten. Die Begriffe ausreichend, zweckmäßig und wirtschaftlich werden in der Medizin immer mehr in den Vordergrund rücken, ihre Interpretation und die daraus resultierenden Begrenzungen werden die Unfallversicherungsträger für die Zukunft fest-

\section{Physiotherapy after injury. Cost-benefit analysis: how long should physiotherapy be prescribed? - Opinion 2}

\section{Abstract}

Like other concerns, the insurance companies providing legally required accident cover are also considering how the resources they have available could be used in a more precisely targeted and efficient manner. With falling contribution income together with the high unemployment figures and the fall in income resulting from these, such a review is also an urgent necessity. The terms 'adequate','appropriate' and 'economically supportable' will be used with increasing frequency in medicine, and their interpretation and the resulting restrictions will have to be key in determining the policies followed by accident insurance companies in the future. Compared with the legen müssen. Gegenüber den gesetzlichen Krankenkassen haben sie den Vorteil der weitergehenden physikalischen Maßnahmen in der stationären Rehabilitation, der unbürokratischeren Wiedereingliederungsverfahren, dem Berufshelfersystem sowie dem Grundprinzip Heilung vor Rente. Nichtsdestotrotz wird auch im UV-System auf Dauer eine Mengenbegrenzung nicht zu umgehen sein.

\section{Schlüsselwörter}

Kostenreduzierung $\cdot$ Mindereinnahmen · Ausreichend $\cdot$ Zweckmäßig $\cdot$ Wirtschaftlich health insurances providing the illness cover required by law they have the advantage of more extensive physical treatments that can be applied during in-patient rehabilitation, nonbureaucratic reintegration procedures, a system of professional helpers, and the basic principle of recovery in preference to pensions. Nonetheless, in the long run the accident insurance companies will also be unable to avoid restricting the amount of treatment prescribed.

\section{Keywords}

Cost savings - Reduced income Adequate . Appropriate - Economically supportable 
digen Krankenkasse zur Genehmigung vorzulegen.

In der Heilmittelverordnung ist $\mathrm{z}$. B. geregelt:

Maximale Verordnungsmengen bei Erst- und Folgeverordnungen sind

- in der physikalischen Therapie: bis zu 6

- in der Ergotherapie bis zu 10 Einheiten.

Es werden folgende therapeutische Maßnahmen unterschieden:

- Maßnahmen der physikalischen Therapie

- Erkrankungen der Stütz- und Bewegungsorgane

- Erkrankungen des Nervensystems

- Erkrankungen der inneren Organe

- Sonstige Erkrankungen

- Maßnahmen der podologischen Therapie

- Maßnahmen der Stimm-, Sprechund Sprachtherapie

- Maßnahmen der Ergotherapie

\section{Gebot der Wirtschaftlichkeit}

Hierzu müssen folgende Punkte abgeklärt werden:

- Kann das Behandlungsziel durch eigenverantwortliche Maßnahmen des Patienten erreicht werden?

- Benötigt man überhaupt eine Heilmittelverordnung?

- Kann nicht durch Verordnung eines Arzneimittels ebenfalls eine Verbesserung der Einschränkung erreicht werden?

Der Vertragsarzt muss prüfen, ob unter Abwägung der jeweiligen Therapierisiken das Behandlungsziel qualitativ gleichwertig und kostengünstiger erreichbar ist. Die gleichzeitige Verordnung mehrerer Heilmittel ist nur dann ausreichend, zweckmäßig und wirtschaftlich, wenn dadurch ein therapeutisch erforderlicher Synergismus erreicht wird.

\section{Fazit}

Unter dem Eindruck rückläufiger Einnahmen und Beiträge auch bei den Unfallversicherungsträgern, durch hohe Arbeitslosenzahlen, Geschäftsaufgaben so- wie Einstieg in die Schwarzarbeit ist es bereits im Gesundheitssystem in allen Bereichen zu finanziellen Mindereinnahmen gekommen. Dies ist bzw. wird auch bei den Unfallversicherungsträgern der Fall sein.

Die Begriffe ausreichend, zweckmäßig und wirtschaftlich werden immer mehr in den Vordergrund rücken, ihre Interpretation und die daraus resultierende Finanz- und Mengenbegrenzung werden die Unfallversicherungsträger, vielleicht auch mit Seitenblick auf das oben beschriebene GKV-System, selbst festlegen müssen. Der Vorteil der Unfallversicherungsträger besteht in weitergehenden physikalischen Maßnahmen, wie stationärer Rehabilitation, unbürokratischeren Wiedereingliederungsverfahren, Berufshelfern sowie dem Grundprinzip: Heilung vor Rente. Nichtsdestotrotz wird auch im UV-System eine Mengenbegrenzung auf Dauer nicht zu umgehen sein.

\section{Korrespondierender Autor}

Dr. D. Haack

Eierstraße 46

70199 Stuttgart

Haack-Kerber@t-online.de

Interessenkonflikt. Es besteht kein Interessenkonflikt. Der korrespondierende Autor versichert, dass keine Verbindungen mit einer Firma, deren Produkt in dem Artikel genannt ist, oder einer Firma, die ein Konkurrenzprodukt vertreibt, bestehen. Die Präsentation des Themas ist unabhängig und die Darstellung der Inhalte produktneutral. 